\title{
Kernos
}

Revue internationale et pluridisciplinaire de religion grecque antique

1 | 1988

Varia

\section{Simulacre humain et offrande rituelle}

\author{
Éveline Loucas-Durie
}

URL : http://journals.openedition.org/kernos/107

DOI : $10.4000 /$ kernos. 107

ISSN : 2034-7871

\section{Éditeur}

Centre international d'étude de la religion grecque antique

\section{Édition imprimée}

Date de publication : 1 janvier 1988

ISSN : 0776-3824

\section{Référence électronique}

Éveline Loucas-Durie, « Simulacre humain et offrande rituelle », Kernos [En ligne], 1 | 1988, mis en ligne le 31 janvier 2011, consulté le 06 mai 2019. URL : http://journals.openedition.org/kernos/107 ; DOI : 10.4000/kernos. 107 


\section{SIMULACRE HUMAIN ET OFFRANDE RITUELLE}

Lorsque vous parlez de simulacre d'être humain dans le cadre de la Grèce antique, le type d'objet que généralement votre interlocuteur imagine aussitôt, ce sont ces nombreuses statues qu'elle nous a laissées, principalement en pierre et en bronze. Ces objets nous sont familiers, puisqu'ils ont subsisté jusqu'à nos jours, mais surtout parce que cette forme de représentation a profondément marqué la civilisation de l'Europe. Les statues de l'antiquité grecque sont, comme on l'entend généralement, des effigies autonomes d'être humain, d'une certaine grandeur, exécutées dans une matière noble, sinon précieuse, et pour lesquelles les Anciens n'avaient pas de terme générique analogue ${ }^{1}$. Elles ont directement attiré l'attention par leur aspect. Mais on en vint aussi à s'interroger sur la signification et la fonction qu'elles avaient pour les Anciens à des périodes définies, segments temporels à la distinction desquels elles ont souvent contribué, précisément en vertu des conventions esthétiques qu'elles reflètent.

Ainsi, par exemple, J. Ducat ${ }^{2}$ a étudié la pluralité de destination de la figure uniforme du kouros, type canonique de la Grèce archaïque. Hormis sa fonction de statue de culte au quel cas il est un substitut de la divinité, lorsqu'il était élevé sur une tombe, le kouros était le substitut d'un mort; mais encore, consacré en offrande dans le sanctuaire d'une divinité, il remplace un être vivant, en tant que sub-

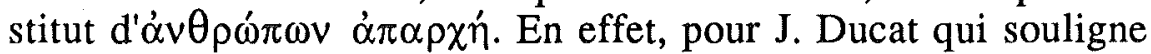
une réalité qui n'est plus directement perçue aujourd'hui par tous ceux qui participent de la civilisation européenne, à savoir que la statue d'un homme n'est «pas [...] un objet «en soi» tel qu'un trépied par exemple, mais [...] une représentation qui renvoie inmanquablement à ce dont elle tient la place ${ }^{3}$ », une offrande de cette

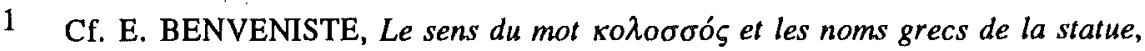
in $R P h$ (1932), p. 118-135.

2 J. DUCAT, Fonctions de la statue dans la Grèce archaique : kouros et kolossos, in $B C H, 100(1976)$, p. 239-251.

3 Ibid., p. 242. 
nature procède de la notion de substitution, non pas par une modification de l'espèce de la victime, alors dans un contexte sacrificiel, mais par une modification du rite lui-même : «la victime reste l'homme, mais le sacrifice est remplacé par la consécration ${ }^{4} »$.

Cette notion de substitution, que J. Ducat a discernée en cherchant à définir les fonctions de la statue dans la Grèce archaïque au départ d'une sorte d'objet, ledit kouros, et à travers un mot, kolossos, est présente également dans d'autres simulacres d'être humain, difficilement - sinon en aucune manière - qualifiables de «statues», impliqués dans diverses cérémonies qu'étudie plus particulièrement celui qui s'intéresse à la religion. Cependant, les obstacles que le chercheur rencontre sont multiples : ces simulacres sont de nature variée, les sources qui révèlent leur utilisation, diverses, et dans certains cas, même, elles sont si peu explicites que la réalité du simulacre est uniquement supposée; et encore faut-il pouvoir préciser de qui exactement ces simulacres sont le substitut, ce qui dépend du contexte rituel souvent malaisé à définir.

Voilà ce que nous soulignerons au fil d'exemples dont l'ordre de présentation n'a pas été imposé par une quelconque hiérarchie des disciplines auxquelles ils ont été déjà rattachés en vertu de l'un ou l'autre de leurs caractères et qualités, ni dicté par les critères d'examen relevant d'une de ces disciplines; il a été suggéré par les affinités qu'ils peuvent présenter les uns avec les autres, et qui ont été reconnues par ceux qui se sont appliqués à approfondir la connaissance des croyances, des idées et des pratiques religieuses de la Grèce antique.

Un de ceux-ci, et non des moindres, M. Nilsson, regroupant nombre de manifestations collectives à caractère cérémoniel se signalant par un grand feu, estimait retrouver en Grèce antique la coutume, répandue en différents points du globe et à diverses époques, du fire festival, à l'occasion duquel, dans certains cas, des

4 Ibid., p. 244. 
effigies étaient jetées dans les flammes ${ }^{5}$. Parfois, ce n'est plus perceptible qu'au travers de données mythologiques, comme le châtiment que Talos inflige au passant capturé tel que l'interprète le savant suédois ${ }^{6}$. Cette pratique revêtait en chaque lieu un aspect particulier.

Ainsi en irait-il de la fête des Elaphebolia à Hyampolis de Phocide, célébrée, aux dires de Plutarque ${ }^{7}$, en l'honneur d'Artémis et en commémoration de la victoire des Phocéens sur les Thessaliens, si l'on en croit la reconstitution avancée par M. Nilsson ${ }^{8}$. Les Phocéens amoncelleraient, sur un bûcher qu'ils allumeraient ensuite, des biens de toute espèce ainsi que des mannequins qui figureraient les images divines des sanctuaires et la population humaine, parce que la fête reproduirait périodiquement un épisode significatif de la guerre opposant Phocéens et Thessaliens, raconté avec quelques variantes par Pausanias, Plutarque ${ }^{9}$.

Dans un accès de désespoir, les Phocéens, en effet, prirent la décision extrême de ne rien laisser aux mains de l'adversaire en cas de défaite. Aussi rassemblèrent-ils dans un même lieu et à proximité de fagots les femmes et les enfants - qui, consultés, auraient formulé leur consentement - ainsi que toute la richesse mobilière des maisons privées et des édifices publics sous la garde d'hommes. Ceux-ci avaient l'ordre, si leur parvenait la mauvaise nouvelle d'une défaite, de mettre le feu aux fagots et de périr eux-mêmes sur le bûcher ou au combat. Toutefois la victoire évita aux Phocéens cet holocauste. Or, selon M. Nilsson, cet holocauste, paradoxalement, serait réellement effectué aux Elaphebolia, avec des simulacres parmi lesquels devraient se distinguer d'une quelconque manière les images divines d'une part, et de l'autre, les victimes humaines potentielles qui appartiennent au moins à deux classes d'âge et aux deux sexes.

5 Cf. M.P. NILSSON, Fire-Festivals in Ancient Greece, in JHS, 43(1923), p. 144-148; cf. aussi ID., Geschichte der griechischen Religion, I2, 1955, p. $130-132$.

6 ID., Fire-Festivals..., p. 148; A.B. COOK (Zeus, A Study in Ancient Religion, I, Londres, 1914, p.723-724 et sq.), par exemple, a mis la légende en rapport avec la technique de la fonte du bronze à cire perdue.

7 PLUT., De mul. uirt., 244 e.

8 Cf. M.P. NILSSON, Griechische Feste, Leipzig, 1906, p. 221-225; ID., FireFestivals..., p. 144.

9 PAUS., X, 1, 2-9; PLUT., De mul. uirt., 244 b-e. 
Cependant, l'utilisation du simulacre aux Elaphebolia d'Hyampolis est supposée par M. Nilsson dans une tentative problématique de reconstitution de la fête au départ d'un événement qui, certes, participe du serment-ordalie à l'occasion duquel il était parfois fait usage de simulacres-substituts. On se souviendra, par exemple, des kolossoi de cire façonnés et brûlés par la population mixte et de tout âge de Théra, avant le départ de ceux d'entre eux qui deviendront les fondateurs de Cyrène, d'où provient l'inscription du IVe s. av. J.-C. qui en reproduit le serment ${ }^{10}$.

La coutume de brûler une effigie dans un bûcher aurait aussi existé sur le mont Oeta, et la mort d'Héraclès en serait l'aition"1, d'autant plus qu'une fouille archéologique y a dégagé une vaste étendue de résidus sacrificiels calcinés, parmi lesquels furent exhumés des tessons de céramique à vernis noir portant une dédicace à Héraclès et deux statuettes archaïques en bronze bien conservées qui figureraient le héros ${ }^{12}$. Il n'en fallait pas plus aux yeux de $\mathrm{M}$. Nilsson pour y trouver confirmation du fait que l'effigie d'Héraclès y était périodiquement brûlée ${ }^{13}$, comme l'aurait laissé entendre TiteLive ${ }^{14}$; et, toujours selon le savant suédois, ce serait au rite même que pourraient sé référer des représentations de vases attiques ${ }^{15}$. Certes, comme sur la pelikè de Munich $n^{\circ} 2630$ et le cratère en cloche de la collection Mustilli à Santa Agata dei Goti, tous deux du IVe s. av. J.-C. ${ }^{16}$, une scène de bûcher associée à l'entrée d'Héraclès dans l'Olympe est localisée sur l'Oeta. Si M. Nilsson avait raison sur tous les points, il serait intéressant de confronter le type des effigies

10 Cf. Fr. CHAMOUX, Cyrène sous la monarchie des Battiades, Paris, 1953 (BEFAR, 177), p. 105-111.

11 Cf. M.P. NILSSON, Der Flammentod des Herakles auf den Oite, in AfRW, 21(1922), p. 310-316; ID., Fire-Festivals..., p. 144-145.

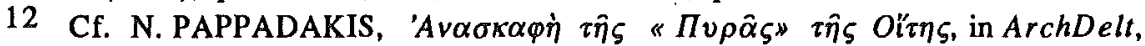
1919, paratt., p. 25-33; cf. aussi ID., in $B C H, 44(1920)$, p. 392-393.

13 NILSSON, Der Flammentod..., p. 315; ID., Fire-Festivals..., p. 144, n. 5; cf. aussi L.R. FARNELL, Greek Hero Cult and Ideas of Immortality, Oxford, 1921, p. 166-169, et à ce propos, vide Fr. FRONTISI-DUCROUX [op. cit. (infra n. 18), p. 209]. - M. NILSSON (Der Flammentod..., p. 315) avance même, sans que cela semble assuré, que des êtres humains y ont été brûlés.

14 TIT. LIV., Hist. Rom., XXXVI, 30.

15 Cf. NILSSON, Der Flammentod..., p. 316.

16 Cf. H. METZGER, Les représentations dans la céramique attique du Ve siècle, Paris, 1951 (BEFAR, 172), p. 210-211, pl. XXII et XXVIII. 
d'Héraclès retrouvées sur le site et leur figuration sur les vases. Précisément sur les exemples cités on peut voir un tronc humain acéphale, qui prend singulièrement l'allure d'une cuirasse sur la pelikè de Munich. Mais pour ce qui est de ces représentations, on ne peut d'office conclure à un contexte rituel, surtout que les images céramographiques ont leur propre code de lecture ${ }^{17}$. En outre, le statut mi-humain mi-divin d'Héraclès invitait à préciser ce que figurait son effigie, si elle était effectivement brûlée. Ce serait en fait le corps mortel du héros qu'elle représenterait, car il semble que l'étape nécessaire à son élévation au rang des dieux fût la destruction de sa dépouille charnelle ${ }^{18}$.

La difficulté de discerner la nature divine ou humaine du simulacre se pose à nouveau à qui cherche à expliquer la fête béotienne des Daidala au cours de laquelle intervenaient sûrement des simulacres, comme le rapporte Pausanias ${ }^{19}$.

La fête des Daidala, célébrée périodiquement et en deux temps, devait son nom à des effigies confectionnées dans le bois d'un chêne choisi selon une procédure définie et parées en nymphai, qui étaient aussi appelées xoana. Et puisque ces figures étaient destinées à être complètement détruites à l'occasion d'un sacrifice à Zeus et à Héra avec diverses victimes animales dans les flammes d'un autel-bûcher érigé sur le mont Cithéron, M. Nilsson a également compté cette fête parmi les exemples grecs antiques de bon fire ${ }^{20}$.

Le mythe se rapportant à cette célébration, raconté par Pausanias et Plutarque chez Eusèbe ${ }^{21}$, disait grosso modo que Zeus reconquit l'amour d'Héra en feignant de se marier avec une image de bois revêtue d'un voile, censée être Plataia, fille d'Asopos (Pausanias) ou

17 Cf. en demier lieu, A. VERBANCK-PIÉRARD, Images et piété en Grèce classique: la contribution de l'iconographie céramique à l'étude de la religion grecque, ici même, où l'on trouvera une bibliographie abondante.

18 Cf. Fr. FRONTISI-DUCROUX, Dédale. Mythologie de l'artisan en Grèce ancienne, Paris, 1975, p. 210.

19 PAUS., LX, 3.

20 NILSSON, Fire-Festivals..., p. 144; cf. aussi ID., Feste, p. 50-56, ID., Geschichte, $\mathrm{I}^{2}$, p. 131.

21 PAUS., IX, 3; PLUT. in EUSEB., Ev. Prep., III, 1, 6 (en III, 1, 3 est rapporté un autre mythe qui n'est pas mis directement en relation avec la fête. 
Daidalè (Plutarque). L'opinion que les daidala utilisés lors du rituel figuraient Héra, voire même Zeus aussi, a eu largement cours dans les différentes explications avancées ${ }^{22}$. Cependant, pour W. Furley ${ }^{23}$, qui prend en considération non seulement le mythe tel qu'il est associé à la fête par Pausanias, mais aussi le contexte plus général des traditions régionales comme elles sont rapportées par le même auteur, le daidalon figure une divinité topique, autochtone, ancêtre de la population locale: Plataia, la nymphe-arbre de chêne. Fr. Frontisi-Ducroux ${ }^{24}$, pour sa part, s'en tenant aux données du mythe, a défendu que ces daidala étaient des fausses femmes humaines offertes à Zeus, des substituts de fiancées, et, poussant plus loin encore l'interprétation, des substituts d'anciennes victimes humaines.

Dans le cadre de cette dernière hypothèse, les parallèles s'imposent à l'esprit : les mannequins d'osier des Argées romaines tels que les considéraient les Anciens eux-mêmes ${ }^{25}$, ou cet eidôlon substitué in extremis à Iphimédée ${ }^{26}$, autre nom d'Iphigénie, quoique dans ce dernier cas il ne soit pas clair s'il est question d'un double immatériel, une espèce de fantôme, ou d'une image tangible dont l'aspect pouvait être des plus divers. Ces trois exemples (les Daidala, les Argées et le sacrifice d'Iphimédée) posent également, outre la question de la signification du simulacre dans le rite, le problème de préciser celle qu'il a dans le mythe, puisqu'il existe aussi à ce niveau-là, mais cela relève donc, d'une manière plus générale, de l'essence même du mythe et du rapport qu'a ce dernier avec le rite.

Simulacre-substitut d'ancienne victime humaine, c'est encore une hypothèse que peut être amené à soutenir le chercheur, à la suite d'une analyse de l'ensemble des documents à sa disposition. Une pareille démarche en commun avec notre époux nous autorisait, nous semblait-il, à proposer une telle interprétation des statuettes zoocéphales en terre cuite découvertes par dizaines dans les cendres

22 Cf., pour une revue sommaire des diverses théories, FRONTISI-DUCROUX, op. cit., p. 197-199.

23 W.D. FURLEY, Studies in the Use of Fire in Ancient Greek Religion, New York, 1981, p. 202-209.

24 FRONTISI-DUCROUX, op. cit., p. 206-212.

25 Cf. VARR., De ling. lat., VII, 44; OVID., Fasti V, 621-662; DION. HAL., Ant. rom., I, 38, 2.

26 HES., fr. 23 a Merkelbach-West. 
sacrificielles du megaron (sorte d'autel monumental) au sanctuaire de la divinité orgiastique Despoina à Lykosoura, et datées à l'époque hellénistique et romaine ${ }^{27}$. Ces statuettes sont hautes de 15 à $20 \mathrm{~cm}$ et reflètent plusieurs types distingués entre autres par le moule utilisé pour leur fabrication. Mais en règle générale elles montrent un corps humain enveloppé jusqu'aux pieds dans un long manteau et surmonté d'une tête d'animal, tantôt d'ovin, tantôt de bovidé. Comme nous l'avons développé ailleurs ${ }^{28}$, nous considérons que ces statuettes répondent à la mention d'agalma que prescrit d'utiliser pour le sacrifice la loi sacrée (datée du IIe s. av. J.-C.) et qu'elles représentent les fidèles en costume cérémoniel dont l'élément essentiel est le masque animal, tels qu'ils sont figurés également sur la bordure du manteau provenant de la statue cultuelle de Despoina.

Certaines statuettes sont gravées au revers d'un nom qui se retrouve de l'une à l'autre et que l'on suppose généralement être la marque du coroplathe. Pour en être certain, un réexamen du matériel s'avère pour le moins indispensable. Si cette hypothèse se vérifie alors, on remarquera le jeu complexe de relations entre le fabricant de l'objet, l'usager et la divinité à laquelle il est destiné, relations qui, cependant, ne s'expliqueront peut-être pas uniquement sur le plan religieux.

Il est néanmoins vrai que pour certaines occasions, surtout lorsque la cérémonie concernait des personnes précises, l'individualisation du'simulacre-substitut peut avoir été recherchée. Dans le cadre de vestiges matériels, ce sont même le type d'objet retrouvé et le lieu de leur découverte qui semblent le suggérer.

Les fragments d'effigies mis au jour dans un tumulus (tombe $n^{\circ} 77$ ) aux confins sud-ouest de la nécropole de Salamine de Chypre illustreraient cette situation ${ }^{29}$; il s'agit plus exactement d'un céno-

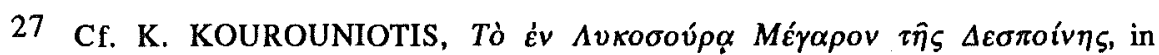
ArchEph (1912), p. 142-161, et surtout fig. 23-34. Cf. aussi

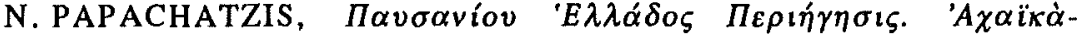
'A $\rho \kappa \alpha \delta ı \kappa \dot{\alpha}, 3$, Athènes, 1980, p. 331-341; M. JOST, Sanctuaires et cultes d'Arcadie, Paris, 1985 (Et. Pélop., 9), p. 177 et pl. 45, fig. 3-4.

28 I. LOUCAS et E. LOUCAS-DURIE, La mention du mot AГA $\Lambda$ MA dans la loi sacrée IG V 2, 514 de Lykosoura, in Peloponnèsiaka, 16(1985), p. 297-314.

29 V. KARAGEORGHIS, Chronique des fouilles et des découvertes archéologiques à Chypre en 1966, in BCH, 96(1967), p. 328-337; ID., Salamis in Cyprus. Homeric, Hellenistic and Roman, Londres, 1969, p. 151-164, fig. 92-97, 
taphe, daté de la fin du IVe s. av. J.-C., dont l'érection s'est accompagnée d'une cérémonie au cours de laquelle des offrandes ont été brûlées au coeur d'un bûcher allumé au centre d'une plateforme en briques de pisé. Les effigies, qui auraient été au nombre de seize et auraient figuré, presque grandeur nature, des hommes et plus d'une femme, avaient été façonnées en argile crue appliquée et retenue (dit le fouilleur, V. Karageorghis) par des clous en fer sur des pieux de bois disposés symétriquement en quadrilatère sur la plateforme. Elles devaient être parées de bijoux et d'autres accessoires, et drapées dans un vêtement dissimulant la totalité du corps à l'exception des extrémités. Les pieds, les mains et les têtes étaient en effet soigneusement modelés. Sur les cinq têtes retrouvées, il y en a une de femme et deux de jeunes gens, à la physionomie assez idéalisée, ainsi que deux d'hommes âgés aux traits fortement marqués. Ces dernières auraient été des répliques de véritables portraits.

H. Schaefer ${ }^{30}$ a parlé de portrait aussi pour cet eidôlon de roi, dont fait part une source écrite, auquel les Spartiates ont rendu les honneurs funèbres. Plus précisément encore, après confrontation du témoignage d'Hérodote (VI, 58) avec celui des scholies, il a conclu qu'il s'agissait du portrait de Léonidas dont le corps n'avait pu être rapatrié depuis les Thermopyles et qui fut alors exceptionnellement honoré en effigie. Désignant certainement un objet concret, cet eidôlon qui était censé représenter une personne définie était pour $H$. Schaefer une statue, peut-être même grandeur nature, à laquelle avaient été donnés des traits individuels, plutôt qu'un masque. Cette préférence ne tient néanmoins pas compte de la tradition locale de masques en terre cuite offerts en ex-voto au sanctuaire d'Orthia ${ }^{31}$; et si certains d'entre eux ont atteint la limite de la caricature, d'autres ne sont pas éloignés du portrait ${ }^{32}$. Outre le fait que le mot «statue» ne correspond à rien de précis dans la pensée grecque ancienne ${ }^{33}$, la

pl. XV-XVI; ID., Note sur les tombes royales de Salamine, in RA (1969), p. 57-80, plus précisément p. 75-79; ID., Excavations in the Necropolis of Salamis, III, Nicosie, 1973, 1, p. 198-200, 2, pl. L-P, et CCV-CCXVII.

30 H. SCHAEFER, Das Eidolon des Leonidas, in Charites. Studien zur Altertumswissenschaft, Bonn, 1957, p. 223-233.

31 Cf. R.M. DAWKINS, The Sanctuary of Artemis Orthia at Sparta, in JHS suppl. 5(1929).

32 Cf. J.Ch. BALTY, Portrait grec, portrait romain, in AnnHAArch, 3(1981), p. 47-64, plus précisément p. 57 et pl. I. 1.

33 Cf. par exemple BENVENISTE, art. cit. (supra n. 1); DUCAT, art. cit. (supra n. 2), p. 246. 
différence, à nos yeux en tout cas, n'est pas grande entre un masque rapporté de quelque manière que ce soit à un vêtement pour simuler une silhouette humaine, et une cffigie semblable à celles exhumées à Salamine de Chypre. D'ailleurs, selon l'interprétation de $\mathrm{K}$. Hadjiioannou ${ }^{34}$, l'usage auquel ces simulacres ont été destinés serait identique : les figures d'argile de la nécropole de Salamine auraient reçu les honneurs funèbres à la place des corps eux-mêmes, demeurés introuvables, des victimes du combat opposant les Salaminiens à Démétrius Poliorcète en 307-306 av. J.-C.; des membres de la famille royale de Nikokréon peut-être.

V. Karageorghis, quant à lui, alléguant certains témoignages anciens dont l'exactitude a été contestée ${ }^{35}$, estime pouvoir rapprocher les trouvailles de cette tombe $n^{\circ} 77$ d'un événement qui aurait marqué tragiquement l'histoire de Salamine ${ }^{36}$ : le suicide, en 311-310 av. J.-C., de Nikokréon, accusé d'avoir trahi Ptolémée, et qui entraîna l'extinction de sa maison. En effet, son épouse et, sur l'instigation de celle-ci, les autres parents proches, tandis qu'ils avaient mis le feu au palais, se sont immolés. Les effigies d'argile les représenteraient donc individuellement et la cérémonie funèbre, que les Salaminiens (ou Ptolémée) auraient célébrée pour honorer dûment les disparus par le truchement de simulacres, aurait comporté tout un aspect expiatoire exigé par ces morts violentes.

En parallèle à cette interprétation, on a pu citer un cas de rituel expiatoire avec simulacres rapporté par Valerius Flaccus ${ }^{37}$. Cet auteur raconte qu'à la suite d'une infortune, les Argonautes s'étaient involontairement rendus coupables du meurtre de leurs hôtes de la veille, le roi Cyzique et son peuple, lors d'un affrontement armé. Et bien qu'ils eussent honoré et brûlé les dépouilles, ils demeuraient accablés d'un mal étrange dont ils ne furent libérés qu'après une

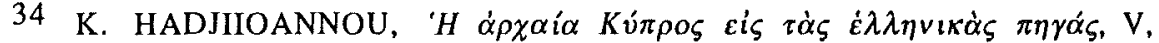
Nicosie, 1983, n ${ }^{\circ} 162$, p. 156-158.

35 HADJIIOANNOU, op. cit., I, 1971, n 77,80 et 81; IV B, 1980, p. 14-15. Cf. aussi H. GESCHE, Nikokles von Paphos und Nikokreon von Salamis, in Chiron, 4(1974), p. 103-125 et, plus spécialement, p. 109-110 et n. 24.

36 KARAGEORGHIS, Chronique..., p. 337; ID., Salamis..., p. 153-164; ID., Excavations... III, p. 201-202. (cf. supra n. 29).

37 VAL. FLACC, Argon., III, 362 sq, plus spécialement 417-455. Cf. FRONTISIDUCROUX, op. cit. (supra n. 18), p. 212 n. 58. 
procédure longue et complexe de purification dirigée par le devin Mopsus. La dernière phase consistait en une incantation adressée aux morts, les priant de mettre fin aux tourments qu'ils infligeaient aux Argonautes, et prononcée devant des simulacres de guerriers. Ceuxci sont uniquement décrits comme des effigies d'hommes confectionnées au départ de troncs de chêne et munies de fausses armes. Ils seraient en nombre égal à celui des Argonautes, selon P. Boyancé, d'après qui ils les représenteraient ${ }^{38}$. On ignore cependant si l'individualisation a été poussée jusqu'au portrait ou si elle s'est arrêtée à la figuration du caractère estimé essentiel, en l'occurrence, la qualité de guerrier. Le nom de trophée (tropaion) a même été avancé pour ces effigies, mais rien n'est moins sûr ${ }^{39}$.

Ces trois cérémonies révélées par des sources tantôt archéologiques tantôt écrites, envisagées ci-dessus, paraissent partager le fait d'avoir été réalisées pour résoudre un problème ponctuel dans le cadre d'un rituel purificatoire et expiatoire. L'histoire de Charilla, si l'on en croit le témoignage de Plutarque ${ }^{40}$, enseigne qu'un rite exceptionnel célébré à des fins expiatoires peut avoir été institué en cérémonie périodique.

Le suicide par vengeance de Charilla, la jeune orpheline dont l'attitude de suppliante n'avait pas été respectée par le roi qu'elle implorait, intensifia encore la disette qui sévissait déjà et obligea les Delphiens à l'apaiser en procédant à un rite sacrificiel joint à une purification, qui consistait à reproduire en quelque sorte ce qui s'était passé et qui était depuis lors répété tous les huit ans. Le roi distribuait aux étrangers et aux citoyens une part de produits agricoles - qu'il avait refusée à la suppliante - tandis qu'était amené un

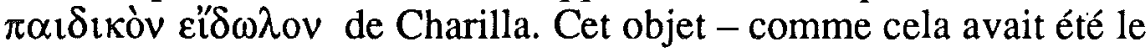
cas pour Charilla en personne - était frappé par le roi puis emporté par la première des Thuyiades dans un endroit inculte. L'objet y était

38 VAL.FLACC., Argon., III, 444-445. Cf. P. BOYANCÉ, Un rite de purification dans les Argonautiques de Valerius Flaccus, in Etudes sur la religion romaine, Rome, 1972 (coll. $E F R, 11$ ), p. 318-345, plus spécialement p. 338-339 $[=R E L, 13(1935)$, p. 107-136].

39 Cf. BOYANCÉ, art. cit., p. 339-340. Sur le trophée en général, avec une révision des théories antérieures, R. LONIS, Guerre et religion en Grèce à l'époque classique, Paris, 1979, p. 129-142.

40 PLUT., Mor., 293 b-f (=Quaest. Gr., 12). 
muni d'une corde au cou et ensuite fiché là-même où avait été enterré le corps de la jeune fille après qu'elle se fut pendue.

C'est à nouveau un individu précis que représente le simulacre. Mais l'aspect en demeure indéfini, si ce n'est que, tout au inoins d'après le témoignage de Plutarque, le caractère enfantin a été souligné. Or un règlement religieux de Cyrène, daté de la seconde moitié du IVe s. av. J.-C. ${ }^{41}$, qui précise le rituel que doit observer le maître de maison accueillant un suppliant venu de l'étranger, semble indiquer, d'après l'interprétation qu'en a proposée J. Servais ${ }^{42}$, que le simulacre pouvait figurer également un individu dent l'identité est justement inconnue.

En effet, le maître de maison est enjoint à appeler nominalement en sommation l'envoyeur du suppliant, mais s'il en ignore le nom, il dira : «Humain, que tu sois homme ou femme !»; il confectionnera ensuite des kolossoi, un masculin et un féminin, en bois ou en terre, qu'il recevra à sa table, et qu'il ira enfin ficher en terre dans un bois inculte. Ces kolossoi en matière périssable auraient été des objets de dimensions réduites, de facture assez grossière, dont le degré de figuration peut se limiter strictement à la distinction des sexes. Cependant ils suffisent pour matérialiser une invocation vague. En fait, comme l'a souligné J. Servais, ces kolossoi ont un rôle

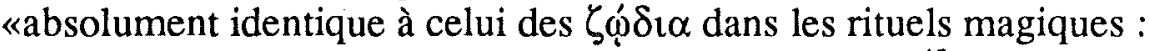
figurer un absent sur lequel on ne peut agir directement ${ }^{43}{ }$.

Ainsi, dans le procédé d'individualisation, le simulacre entre en concurrence avec le nom, et l'un et l'autre procédé a été utilisé dans les pratiques magiques.

Ainsi, les exemples que nous avons envisagés nous ont amenée bien loin de la «statue». Tous ces objets, décrits par nos sources, retrouvés ou «reconstitués», utilisés dans divers contextes, qu'ils

\footnotetext{
41 Cf., pour une présentation critique du passage, avec la bibliographie antérieure, J. SERVAIS, Les suppliants dans la loi sacrée de Cyrène, in $B C H$,

43 Ibid., p. 127.
} 
s'appellent agalma, eidôlon, kolossos, daidalon..., qu'ils soient le fruit d'une exécution raffinée ou grossière, qu'ils montrent l'enveloppe du corps humain dans le détail ou une silhouette schématique, qu'il aient l'apparence d'un portrait ou celle d'une représentation impersonnelle, qu'ils soient façonnés dans une matière précieuse ou vulgaire, résistante ou périssable, tous ces objets sont des simulacres d'être humain qui ont pu lui être substitués. Aux yeux des Anciens, il suffisait, semble-t-il, d'agir avec eux de la même manière qu'avec leurs semblables en chair et en os.

Thisseos 45, Chalandri,

Eveline LOUCAS-DURIE GR - 15234 ATHËNES 